\title{
Comment on "Fast-slow mode coupling instability for coasting beams in the presence of detuning impedance"
}

\author{
Alexey Burov $\odot^{*}$ and Valeri Lebedev $(0$ \\ Fermi National Accelerator Laboratory, Batavia, Illinois 60510, USA
}

(Received 7 April 2021; accepted 8 July 2021; published 29 July 2021)

\begin{abstract}
We show untenability of key points of the recent article of N. Biancacci, E. Metral, and M. Migliorati [Phys. Rev. Accel. Beams 23, 124402 (2020)], hereafter the article and the authors. The main Eqs. (23) are shown to be incorrect even as an approximation. The article claims the solution of this pair of equations to be in "excellent agreement" with the pyHEADTAIL simulations for CERN PS, which is purportedly demonstrated by Fig. 6. Were it so, it would be either a signal of a mistake in the code or a consequence of a nonsmoothness of the simulations.
\end{abstract}

DOI: 10.1103/PhysRevAccelBeams.24.078001

The authors of Ref. [1] suggest a new type of transverse collective instabilities of coasting beams, based on coupling of modes with different longitudinal harmonics, $n_{1} \neq n_{2}$. Their analysis is based on the smooth approximation, i.e., on the linear differential equation for the transverse offset $y(s, t)$ with time- and space-independent coefficients. From a conventional smooth Eq. (22), the authors derive a new Eq. (23) that has nonzero cross-terms, or mode-coupling terms. This derivation is certainly incorrect: when the Fourier transformation over $s$ is applied to the smooth Eq. (22), all the cross-terms necessarily cancel. This cancellation reflects the translational symmetry, provided by the smooth approximation.

To demonstrate this in the context of the article, let us start from its Eq. (22), consistently written in the original $s$-domain, as in its Eq. (3),

$\ddot{y}(s, t)+\omega_{\beta}^{2} y(s, t)=\frac{\left\langle F_{y}^{\text {driv }}\right\rangle(s, t)}{\gamma m_{0}}+2 \omega_{\beta} \Delta \Omega^{\text {det }} y(s, t)$,

where the driving force is conventionally defined by $\mathrm{Eq}(7)$,

$$
\left\langle F_{y}^{\text {driv }}\right\rangle(s, t) \propto \int_{t}^{\infty} W_{y}^{\text {driv }}\left(v t^{\prime}-v t\right) y\left(s, t^{\prime}\right) \mathrm{d} t^{\prime}
$$

Expanding the sought-for eigenfunction of our Eq. (1) into a Fourier series,

\footnotetext{
*burov@fnal.gov

Published by the American Physical Society under the terms of the Creative Commons Attribution 4.0 International license. Further distribution of this work must maintain attribution to the author(s) and the published article's title, journal citation, and DOI.
}

$$
y(s, t)=\exp (-i \Omega t) \sum_{n} y_{n} \exp (i n s / R),
$$

and applying the Fourier transform to the left and right parts of the equation, we get,

$-\left(\Omega-n \omega_{0}\right)^{2} y_{n}+\omega_{\beta}^{2} y_{n}=2 \omega_{\beta}\left(\Delta \Omega_{n}^{\text {driv }}+\Delta \Omega^{\text {det }}\right) y_{n}$,

with no cross-terms.

Another point of our comment relates to the claimed excellent agreement between the theory of Eq. (23) and pyHEADTAIL simulations. In light of incorrectness of the former, this agreement may happen either due to a special nonsmoothness of the simulations or through a correlated mistake in the code. The authors should explain how the lattice smoothness is implemented, what the phase advance per cell is, how many cells per ring and how many impedance kicks per turn there are, and how the claimed agreement depends on these parameters. If the code is correct, the smoother the lattice is and the denser the impedance kicks are, the weaker the mode coupling must be. The simulation results should be checked in this respect, to make sure that the code works correctly. If it does, the claimed agreement with Eq. (23) will disappear.

This manuscript has been authored by Fermi Research Alliance, LLC under Contract No. DE-AC02-07CH11359 with the U.S. Department of Energy, Office of Science, Office of High Energy Physics.

[1] N. Biancacci, E. Métral, and M. Migliorati, Fast-slow mode coupling instability for coasting beams in the presence of detuning impedance, Phys. Rev. Accel. Beams 23, 124402 (2020) 